\title{
Spinal cord stimulation what do we know?
}

\author{
Volume 4 Issue I - 2016
}

\section{Introduction}

This treatment modality first saw day light due to clinical outgrowth of gate control theory. ${ }^{1}$ It works on the basic principle of electrical stimulation of large fibres in dorsal columns inhibiting nociceptive transmission in smaller Adela and C fibres at segmental level. The first clinical application was shown in 1967 via subarachnoid route by Shelley. In the early days of usage this treatment modality was widely used for chronic pain conditions which included failed back surgery, phantom limb, segmental pain post spinal cord injury and plexus injury as evaluated by. ${ }^{2}$ The initial response was terrific showing almost $50 \%$ reductions in pain for almost $38 \%$ population, it was the long term follow up which showed only $22.8 \%$ patients achieving satisfactory analgesia.

\section{Mechanism of Action: SCS works in multiple domains}

Neurochemical effects: There is no relation between SCS and opioid release, there is an increase dorsal horn GABA expression seen in animal model after SCS stimulation, levels of Substance P and serotonin are shown to be elevated by SCS. Studies have proven that excitatory amino acid release is suppressed by SCS via action on GABA-B receptors and intra-thecal adenosine as well baclofen may potentiate the effect of SCS. The net effect of these neurochemical effects reduces dorsal horn neuronal excitability. ${ }^{3}$

Neurophysiological effects: SCS application modulates nociceptive response via an action on abnormal pain-mediating Abeta fibre function. The response of wide dynamic range of neurons (WDR) to chemical, thermal and mechanical stimuli in animal neuropathic models has shown suppression with application of SCS. Various models have shown specific role played by remote nervous system like anterior pretectal nucleus which modulates the descending pain inhibitory pathways due to SCS stimulation. ${ }^{3}$

Cardiac/Vascular effects: In angina pectoris, SCS has been demonstrated to reduce noxious input to pain pathway, inhibit the afferent sympathetic nervous system and stabilize intra-cardiac neuronal activity. In ischemic pain models SCS is associated with peripheral vasodilation, mediated via the release of calcitonin generelated peptide (CGRP).

\section{Clinical applications}

\section{Neuropathic Pain}

SCS has been used in a wide range of neuropathic pain conditions which have shown promise, pain relief in order of $50 \%$ has been demonstrated in conditions including, FBSS, CRPS, Peripheral nerve and plexus injuries, Segmental pain post spinal cord injuries and post amputation pain. ${ }^{4}$ Case control studies suggest response rates of $12 \%$ - $88 \%$ for FBSS. A review of 39 case control studies over a 28 year period demonstrated 59\% response rate (range:15-100\%), overall complication rates were around $42 \%$ mainly related to SCS systems. Most of studies report insufficient evidence to draw conclusions about the efficacy of SCS relative to other therapies, despite that there is evidence to suggest that majority of patients treated with SCS for FBSS are satisfied and would recommend this to others suffering

\author{
Apurv Mahalle, Madhur Chadha \\ IPM Consultant pain medicine Acharya Vinobha Bhave Hospital \\ Sawangi Wardha, India
}

Correspondence: Apurv Mahalle, FIPM Consultant pain
medicine Acharya Vinobha Bhave Hospital Sawangi Wardha, India, Email drapurvm@gmail.com

Received: May 25, 2015 | Published: January 07, 2016

from same condition. ${ }^{5}$ North and Wetzel ${ }^{4}$ suggested that SCS may be superior to re-operation in FBSS; the evidence as such is limited. A systematic review of SCS in FBSS and CRPS by Turner ${ }^{6}$ reported mild to moderate improvement with SCS. A similar review by Frey ${ }^{7}$ concluded a limited to moderate evidence for long term pain relief in patients with FBSS. The combination of CBT, Physiotherapy and a multimodal approach targeting bio-psycho-social formulations for management in all chronic neuropathic pain conditions are showing better results along with somatic interventions as pointed out by Esmer $^{8}$

\section{Peripheral vascular disease}

SCS was first utilized in management of PVD in 1976. Multiple retrospective studies have demonstrated efficacy in terms of improved exercise, tolerance, reduced pain and limb salvage. ${ }^{9}$ The selection criteria for this therapy in PVD include peripheral trans-cutaneous oxygen tension with those $>20 \mathrm{mmHg}$ (sitting) having the chance of adequate response. . $^{910}$

\section{Microvascular ischemia}

The role of SCS in micro vascular ischemic syndromes remains unclear, few case series evidence suggesting benefit in diverse range of conditions including Reynaud's.

\section{Refractory angina pectoris}

It is an effective modality since 1985 for refractory angina pain; SCS has shown to reduce the frequency of angina attacks, nitroglycerine consumption and even the total duration of hospitalization and cost of care.

\section{Refractory visceral/abdominal pains}

There is some evidence of use of SCS in cases of refractory mesenteric ischemic pain. ${ }^{11}$ Krames et al. ${ }^{12}$ showed positive responses in IBS with reduction in loose motions and initial reduction of pain with use of SCS.

\section{Newer area of interest}

A recent study conducted by Cecile, ${ }^{13}$ showed promising results in diabetic neuropathy with reduction in pain intensity and the pain becoming more manageable with improved quality of life. 


\section{Miscellaneous conditions}

Stimulation of sacral nerves, specifically S3 has shown results for urinary incontinence secondary to detrusor hyper relfexia, interstitial cystitis, faecal incontinence and in refractory pelvic pain conditions. ${ }^{14}$ The selection criteria and methodology of implantation is addressed more elaborately in specific literature.

\section{Complications and adverse effects}

SCS is a grey area and the failure rates are the blind side which is inconsistently defined. The complications can be defined as failure to gain analgesia despite adequate para-esthesias. This is the basis for temporary screening where $11-25 \%$ population fails permanent implantation. Despite screening and selection for who those gain benefit, analgesic failure can develop over time after a period of successful implantation and is referred to as accommodation. The complication rates from therapy have been more consistently reported and included in a systemic review. ${ }^{6}$ The mean rate of complications across studies was $34 \%$, with $23 \%$ requiring stimulator revision, $11 \%$ removal and $4.6 \%$ infection rate (only $0.1 \%$ deep infection). Of note is a rate of $5.8 \%$ reported for pain in the region of stimulator components. In addition $2 \%$ had dural puncture risk and PDPH episodes. Reasons for need to revise the SCS included lead migration, loss or movement of coverage outside painful area, lead fracture, painful generation and impulse generator exhaustion in a fully implantable systems.

\section{Conclusion}

There is an urgent need for randomized, controlled, long term studies on efficacy of SCS in large patient population. Pain behaviours have been identified as major negative determinants for invasive SCS implantation in a similar fashion poor pacing, fear avoidance, unrealistic expectation and catastrophizing attitude need to be adequately addressed in order to achieve not just a reduction in pain but also an improved functional restoration. The advanced techniques such SCS must not be considered purely "curative", but must be rationally utilized in context of a complete multimodal bio-psychosocial approach which addresses all chronic pain issues and improve the overall quality of life and functionality of a patient.

\section{References}

1. Melzack, Wall. Pain mechanisms: a new theory. Science. 1965;150(3699):971-979.
2. Karnick JU, Thoden U. Spinal Cord Stimulation: Textbook of Pain (2nd edn). Wall PD, Melzack (Eds.), Churchill Livingston, New York, USA, 1989. p. 924.

3. Oakley JC, Prager JP. Spinal Cord Stimulation: Mechanisms of Action. Spine. 2002;27(2):2574-2583.

4. North RB, Wetzel Ft. Spinal cord stimulation for chronic pain of spinal origin: A valuable long term solution. Spine. 2002;27(22):2584-2591.

5. Block AR, Ohnmeiss DD, Guyer RD, et al. The use of presurgical psychological screening to predict the outcome of spine surgery. Spine J. 2001;1(4):274-282.

6. Turner JA, Loescer JD, Sanders SB. Spinal Cord stimulation for patients with failed back surgery syndrome or complex regional pain syndrome: A systematic review of effectiveness and complications. Pain. 2004;108(1-2):137-147.

7. Frey ME, Manchikanti L, Benymain RM, et al. Spinal cord stimulation for patients with failed back surgery syndrome: a systematic review. Pain Physician. 2009;12(2):379-397.

8. Esmer G, Blum J, Rulf $\mathrm{J}$, et al. Mindfulness based stress reduction for failed back surgery syndrome: a randomised controlled trial. $\mathrm{J} \mathrm{Am}$ Osteopath Assoc. 2010;110(11):646-652.

9. Tide JM, Huntoon MA. Review of Spinal cord stimulation in peripheral arterial disease. Neuromodulation. 2004;7(3):168-175.

10. Simpson BA. Spinal cord stimulation in peripheral vascular disease. Electrical stimulation and relief of pain. Pain Research And Clinical Management. 2003;15:131-142.

11. Ceballos A, Cabezudo L, Bovaira M, et al. A possible therapeutic alternative for chronic mesenteric ischemia. Pain. 2000;87(1):99-101.

12. Krames ES, Mousad DG. Spinal Cord Stimulation reverses pain and diarrhoea episodes of irritable bowel syndrome: A case report. Neuromodulation. 2004;7(2):82-87.

13. Cecile De Vos. New Treatment option for pain as a result of Diabetes Mellitus, Diabetic Neuropathic Pain. International Doctoral Research programme thesis article, University of Twente Research institute, Netharland. 2013.

14. Petit P, Thompson J, Chen A. Sacral neuromodulation: New application in treatment of female pelvic floor dysfunction. Current opinion in obst and Gynaec. 2002; 14(5):521-525. 